\title{
PROPOSICIONES PARA LA FORMACIÓN EN LIDERAZGO EDUCACIONAL
}

\author{
Paulo Volante* \\ Alejandro Díaz* \\ Oliver Tapia*
}

* Facultad de Educación de la Pontificia Universidad Católica de Chile. 


\section{RESUMEN}

Actualmente se han intensificado las altas expectativas y los requerimientos en torno al rol que deberían jugar los directores, administradores y otros responsables de la conducción de organizaciones formativas. En este sentido, tanto la investigación internacional -en particular en la tradición anglosajona- como el discurso político nacional, se refieren a la necesidad de fortalecer las capacidades y desempeños del "liderazgo educativo". Por ello, en este artículo se discuten algunos conceptos claves de este rol. Luego se expone la relación entre modelos de formación y estándares internacionales en la materia. Y por último se concluye con algunas recomendaciones en torno al diseño de estos programas en el contexto nacional. Particularmente importantes son las proposiciones de utilizar un enfoque de redes, una óptica interdisciplinaria y un esquema de autoevaluación en la formulación e implementación de estas mallas curriculares.

\section{ABSTRACT}

At present, high expectations and requirements with regard to the role that should be played by directors, managers and other individuals responsible for conducting educational establishments have risen even more. In this respect, international research-particularly studies conducted according to rules in force in Anglo-Saxon academic circles- and also in the national political discourse, are referring to the need to strengthen the abilities and performance of "educational leadership". Consequently, this paper discusses some key concepts related to the role described above. The article then goes on to describe the relationship between training models and international standards in educational leadership. It concludes with some recommendations related to the design of programs for training educational leaders in the national context. Of special interest are the proposals to use a network approach, an interdisciplinary method and a self-assessment system in the formulation and implementation of these curricular programs. 


\section{PROPOSICIONES PARA LA FORMACIÓN EN LIDERAZGO EDUCACIONAL}

\section{DISTINCIONES INICIALES SOBRE GESTIÓN, ADMINISTRACIÓN Y LIDERAZGO EDUCACIONAL}

En general, la discusión sobre la formación de directores, gestores y administradores educacionales adolece de precisión en los conceptos aludidos, pues en estos términos predominan connotaciones en función de proyectos y lecturas específicas. Por ello, parece importante iniciar el tema con algunas distinciones al respecto.

El concepto de administración alude a los procesos regulares que suponen labores especializadas de coordinación de rutina dentro de la organización. Toda administración ha emergido de un anterior proceso de gestión, pero la diferencia está en que la administración no cubre necesariamente la toma de decisiones estratégicas, tal como sí lo hacen los procesos de gestión. En ellos, la toma de decisiones está en función de información incompleta y dinámica. En este contexto predomina un clima de incertidumbre, riesgo y efectos de mediano y largo plazo. Al mismo tiempo estas decisiones en su origen pueden hacer emerger un sistema administrativo y provocar inflexiones en el sistema.

Un criterio menos intuitivo para poder apreciar la diferencia entre los procesos administrativos y los de gestión se puede encontrar en conceptos enmarcados en la teoría cibernética. Maragoh Maruyama acuña los conceptos de "morfostasis" y "morfogénesis". Los procesos morfostáticos buscan el mantenimiento de la forma en un sistema dado, reduciendo las desviaciones a través de mecanismos de control -feedback negativo- que intentan mantener la identidad del sistema a lo largo del tiempo. Los segundos, en cambio, son aquellos que 
aumentan la desviación del sistema y producen la creación de nuevas formas (Rodríguez, D. 1991). En este sentido los procesos administrativos se identifican con mecanismos de control morfostáticos y a su vez los procesos de gestión pueden ser identificados regularmente como morfogenéticos. En los procesos administrativos predominarían las retroalimentaciones negativas reductoras de desviaciones. Por el contrario, los procesos de gestión presentan, en general, una mayor cantidad de retroalimentaciones positivas que pueden amplificar las desviaciones dando lugar más fácilmente a situaciones de cambio e incertidumbre.

El liderazgo, en tanto, pertenecería a un plano distinto a aquel en que se ubica la diferencia entre gestión y administración. El liderazgo estaría asociado al procesamiento de flujos informales existentes en la organización, expresándose en variables como la percepción del carisma, el sistema de creencias, las situaciones de clima laboral, los valores y símbolos cotidianos y las contingencias informales. Por supuesto, estos elementos producen resultados que trascienden el plano informal y que pueden llegar a afectar, para bien o para mal, los objetivos planteados por la organización.

Esta definición del concepto de liderazgo se asemeja a la conceptualización del líder educativo planteada por Heifetz (Heifetz, R. 1994). Heifetz distingue en los problemas de la escuela una dimensión técnica o adaptante, la cual se identificaría con cuestiones de administración y de rutina. Los problemas no adaptantes serían aquellos en los que el liderazgo es crucial, es decir, aquellos que requieren cambios en los valores de las personas, sus creencias y comportamientos (Heifetz, R. 1994). Murphy, por su parte, identifica al líder educativo sobre la base del trabajo en "metas valóricas", definiendo para él tres roles claves: administrador moral, educador y constructor de comunidad (Murphy, J. 2002).

Sin embargo, muchos de los perfiles de líder educativo propuestos por estudios más recientes (Gunter, H., Ribbins, P. 2002; Jackson, B., Kelley, C. 2002; Day, C. 2000; Glassman, N., Cibulka, J., Ashby, D. 2002) indican que una de sus características críticas es el buen manejo de gestión formal, lo que exige de su desempeño elementos de administración profesional y operativa. Por otra parte, nos parece que la dicotomía entre énfasis administrativo y "sicosocial" es superada 
por quienes asumen que el liderazgo educacional consiste en superar tensiones entre ambas dimensiones (Day, C. 2000). Por otra parte, es interesante mencionar los eventuales riesgos y problemas de exacerbar el efecto del liderazgo. Así, por ejemplo, Acuña denuncia los peligros del liderazgo efectivo y muestra que este no siempre va asociado a beneficios para las personas y para las organizaciones (Acuña, E. 1994). Un liderazgo fuerte puede generar una obediencia extrema a la autoridad, acciones irreflexivas, baja tolerancia al conflicto en grupo o censuras a las opiniones discrepantes, lo cual se expresa en pérdidas de información para la toma de buenas decisiones.

\section{PRECISANDO EL LIDERAZGO EDUCACIONAL}

Las tendencias culturales de los últimos cincuenta años en gran parte del mundo han presionado por un aplanamiento de las estructuras organizacionales y su desburocratización. Junto a este fenómeno han surgido diversos conceptos que apuntan a describir la distribución de los procesos de decisión por toda la organización-empowerment-y la superación de la disyuntiva entre procesos de planificación y aquellos propiamente operativos. En educación se han enfatizado los conceptos de liderazgo transformacional (Leithwood, K. 1992), liderazgo distribuido (Spillane, J., et al. 2002) y, sobre todo, el sentido del liderazgo participativo. Todas estas connotaciones aluden a un perfil asociado al traspaso de iniciativa, información y responsabilidad en los diferentes actores del sistema.

En Chile, el Ministerio de Educación ha impulsado un proyecto de ley en que se transfieren varios de estos sentidos a las políticas de concursabilidad, selección y evaluación del desempeño de directores de establecimientos educacionales. En Estados Unidos, el Consorcio Interestatal de Acreditación de Líderes Escolares (ISLLC) ha desarrollado parámetros en función de un perfil de liderazgo que define núcleos comunes de conocimientos, actitudes y desempeños que facilitarían el ejercicio de un liderazgo para el logro de escuelas efectivas y el mejoramiento de resultados educacionales (Volante, P. et al. 2002). El modelo de liderazgo que subyace a esta proposición se relaciona fuertemente con los modelos aludidos, y nos sirve para precisar seis énfasis que pueden orientar procesos de formación, selección y evaluación (ver cuadro 1): 
Cuadro $\mathrm{N}^{\circ} 1$

Estándar de definición

\begin{tabular}{|c|l|}
\hline Visión institucional & $\begin{array}{l}\text { El líder educativo promueve el éxito de todos los estudiantes } \\
\text { facilitando el desarrollo, articulación, puesta en práctica y } \\
\text { cultivo de una visión orientada al aprendizaje que es } \\
\text { compartida y apoyada por la comunidad de la escuela. }\end{array}$ \\
\hline Cultura escolar & $\begin{array}{l}\text { El líder educativo promueve el éxito de todos los estudiantes } \\
\text { abogando, consolidando y sosteniendo una cultura escolar y } \\
\text { un programa educacional conducente al aprendizaje del } \\
\text { estudiante y crecimiento del personal docente. }\end{array}$ \\
\hline Operaciones efectivas & $\begin{array}{l}\text { El líder educativo promueve el éxito de todos los estudiantes } \\
\text { asegurando la gerencia de la organización, de las operaciones } \\
\text { ydelos recursos para crear un ambiente de aprendizaje sólido, } \\
\text { seguro, eficiente y eficaz. }\end{array}$ \\
\hline de los actores & $\begin{array}{l}\text { El líder educativo promueve el éxito de todos los estudiantes } \\
\text { colaborando con las familias y los miembros de la comunidad, } \\
\text { respondiendo a los intereses y a las diversas necesidades de la } \\
\text { comunidad, y administrando los recursos con los que cuenta. }\end{array}$ \\
\hline $\begin{array}{c}\text { Vinculación } \\
\text { con el entorno }\end{array}$ & $\begin{array}{l}\text { El líder educativo promueve el éxito de todos los estudiantes } \\
\text { actuando con integridad, imparcialidad y de una manera ética. }\end{array}$ \\
$\begin{array}{l}\text { El líder educativo promueve el éxito de todos los estudiantes } \\
\text { entendiendo, respondiendo e influenciando al contexto } \\
\text { político, social, económico, legal y cultural. }\end{array}$ \\
\hline
\end{tabular}

Este modelo logra centrar el perfil de liderazgo educacional en la función central de la organización educativa y deja la suficiente flexibilidad para adecuar a las diversos contextos la medición específica de ellos. En este sentido, puede ser una buena orientación a la hora de diseñar y evaluar programas de formación.

\section{FORMACIÓN EN LIDERAZGO, ESTÁNDARES Y CONTEXTOS}

Uno de los puntos claves en la formación del liderazgo educativo es la pertinencia entre las capacidades a desarrollar y el contexto en el que se desenvuelven los egresados. Investigaciones internacionales dan cuenta de la preparación de líderes educativos dependiendo de las condiciones y particularidades de los diferentes sistemas en que actúan 
(Leithwood, K., Jantzi, D., Steinbach, R. 2002). Por ejemplo, si se analiza la relación entre el nivel de descentralización y autonomía del sistema en que se desempeñan los directores, se evidencia que esta formación sería distinta de aquella donde priman contextos centralizados, como es el caso de Francia, Alemania, Hong Kong y Singapur, en comparación con casos como Nueva Zelanda y Holanda, donde se ha dado más descentralización, y por último habría que considerar situaciones intermedias, como la de Estados Unidos y la de Canadá.

En general, parece haber una relación entre sistemas más centralizados y modelos de formación más orientados al perfil de administración y viceversa. Pero también se observan casos donde se busca un equilibrio entre estas polaridades, llegándose a esquemas de formación que pueden ser muy buenos ejemplos para nosotros, como los de Inglaterra, Ontario y Nueva Zelanda. Dependiendo de cada contexto, se hace apropiado un tipo de liderazgo directivo transformacional para contextos más descentralizados, o un liderazgo denominado transaccional para contextos más centralizados (Huber, S.G., West, M. 2002).

Profundizando este análisis, en los países de mayor tradición en liderazgo educacional existe cierto alineamiento con modelos de estándares a la hora de definir en qué y cómo se estructuran los programas de formación. Al respecto, Leithwood, Jantzi y Steinbach (2002) comentan en su estudio cinco importantes series de estándares, a partir de los cuales se deducen elementos comunes en distintos contextos y naciones.

En Estados Unidos se encuentran los Estándares de ISLLC, ya citados anteriormente, los cuales fueron desarrollados a partir de 1996. Estos parámetros se establecieron desde "la investigación sobre la productividad de los líderes educacionales y la experticia de los consultados, con dos propósitos: estimular el pensamiento acerca de la calidad de los líderes escolares y proveer datos que ayuden a los tomadores de decisiones estatales a informarse del desempeño de los líderes, como forma de contribuir a la calidad de su función en las escuelas" (Leithwood, K., Jantzi, D., Steinbach, R. 2002). También en Estados Unidos se mencionan los Estándares de Connecticut, elaborados en base a investigación sobre la efectividad de los directores, cuyos procedimientos de recolección de información incluyeron 
principalmente un gran número de focus groups de líderes de escuelas en ese estado. El propósito del desarrollo de esos estándares fue contribuir a implementar mayor calidad en el liderazgo escolar, para así guiar la formación, acreditación y selección de nuevos líderes escolares, con el fin de mejorar el desarrollo profesional y valoración del desempeño de los egresados.

En Queensland, Australia, se creó un marco de estándares con idénticos propósitos. Este marco fue desarrollado en 1997 y se centra en seis roles claves: liderazgo educativo, dirección y gestión, relaciones con las personas y desarrollo de relaciones, cambios transformacionales, consecución de logros y responsabilidad. Para cada rol se especifican las competencias necesarias. Este marco resultó de la investigación de setenta líderes educacionales en sus lugares de trabajo.

En el Reino Unido se desarrollaron los Estándares Nacionales para Headteachers, por la Agencia de Entrenamiento de Profesores en 1998, con el objeto de explicar los propósitos de la implementación de metas para los estudiantes y promover la calidad. En este modelo se define la experticia en términos de conocimiento, habilidades y atributos, y manejo de áreas claves de dirección. Aquí se consideran, por ejemplo, habilidades del líder en el proceso de toma de decisiones, habilidades de comunicación, autogestión, y atributos como autoconfianza y entusiasmo. Las áreas claves específicas del liderazgo incluyen: dirección estratégica y desarrollo de la escuela, enseñanza y aprendizaje, liderazgo y manejo del personal. Asimismo, aspectos como eficiencia y la efectividad en el manejo de los funcionarios y los recursos, y la responsabilidad (Leithwood, K., Jantzi, D., Steinbach, R. 2002).

En Nueva Zelanda, los estándares para los programas de formación de liderazgo se denominan Principal Performance Management. Estos estándares fueron desarrollados en 1998, a través de una consulta cerrada de directores y autoridades, quienes se basaban en la premisa de que el liderazgo y las habilidades de administración y gestión del director tienen un alto impacto sobre la efectividad de la escuela en los procesos de aprendizaje. Los estándares fueron desarrollados para: ayudar a las escuelas a clarificar el conocimiento, habilidades y actitudes que todos los directores deben demostrar; 
mejorar la calidad, el desempeño y la gestión del director; proveer un marco para identificar el desarrollo de las necesidades profesionales de los directores; y, proporcionar una forma de medición del desempeño de la gestión (Leithwood, K., Jantzi, D., Steinbach, R. 2002). Los estándares profesionales para directores están agrupados en seis categorías o dimensiones profesionales, que incluyen liderazgo profesional, dirección estratégica, dirección de equipos de trabajo, relaciones estratégicas, dirección financiera y requerimientos de estatutos y reportes. Además, consideran la sugerencia de que las escuelas pueden buscar adicionalmente otros parámetros para sus contextos particulares o desarrollar indicadores específicos (criterios de desempeño) para ayudar a clarificar qué se espera de los líderes.

A partir de esta síntesis sobre la lógica en el uso de estándares asociados al liderazgo educacional, podemos deducir su evidente similitud y su utilidad a la hora de diseñar e implementar programas de formación. Los principales ejes para extraer lineamientos relevantes a programas o metodologías de evaluación se reconocen en los siguientes puntos:

- La importancia del desarrollo y construcción de una visión centrada en el aprendizaje de todos los estudiantes y compartida por los actores influyentes en este objetivo.

- Una estrategia de trabajo colaborativo en los procesos de planificación estratégica de la organización educativa.

- Reconocimiento de la necesidad de actuar como un administrador efectivo en asuntos financieros y operacionales.

- Construcción de equipos o staffs de trabajo.

- Capacidad para comunicar un modelo ético claro y coherente (tres de los cinco juegos de estándares hacen mención específica a la labor ética e integridad).

- Establecimiento de mecanismos de monitoreo y evaluación del progreso de los profesores y de los niños, así como de la aplicación de estrategias o planes para el mejoramiento escolar.

- Compromiso y uso de los resultados de los tests estandarizados para guiar el currículo y la instrucción.

- Desarrollo y mantención de relaciones con los padres y la comunidad para vincularlos con la vida de la escuela, y establecimiento de comunicación efectiva con los agentes que influyen en la organización. 
Por tanto, los programas de formación de liderazgo pueden ser evaluados en relación a estos ejes, con lo cual se facilita la coherencia entre proceso formativo y evaluación del desempeño. Es decir, con estos conjuntos de factores para el liderazgo aparece un marco mínimo en la adquisición de conocimientos por parte de quienes se capacitan para ejercer la función de directores de organizaciones educativas y, a la vez, se obtienen parámetros generales para construir un modelo de evaluación que está siendo reconocido en diversas latitudes.

\section{FORMACIÓN DE LÍDERES EDUCACIONALES SITUADA Y EN REDES}

Otra dimensión relevante a la hora de pensar la formación de líderes para la educación, consiste en la capacidad de capturar la complejidad del entorno abriéndose a él de modo de descubrir nuevas posibilidades e incorporar la diversidad de elementos relevantes, evitando el enclaustramiento institucional.

Interesa destacar aquí el concepto de redes, en tanto contenido de formación y elemento de diseño curricular respectivo. Se entiende por red a un conjunto de actores que establecen relaciones de intercambio durables y repetidas, y que carecen de una autoridad organizacional legítima para arbitrar y resolver disputas durante el intercambio (Podolny, J., Page, K. 1998). La evidencia empírica muestra cómo la posición de red de una organización influencia su comportamiento y resultados (Powel, W., Koput, K., Smith-Doerr, L. 1996). Las relaciones de red han sido también descritas como "recursos de red" por cumplir un rol facilitador en ciertos entornos e industrias (Ahuja, G. 2000). Por otra parte, existe evidencia que explica la relación positiva entre densidad de redes y beneficios, tal como lo han mostrado Shan, Walker y Kogut (1994).

Gautam Ahuja (2000) en su estudio demuestra empíricamente que los vínculos de una organización con su entorno son determinantes para los subsecuentes outputs de innovación. Ahuja identifica tres elementos estructurales de la red que pueden acarrear beneficios para la organización. Los vínculos directos se refieren a aquella relación inmediata entre una organización y otra. Este tipo de vínculos puede afectar el output innovador de la organización, en cuanto para esta 
implica el acceso a nuevos conocimientos. La organización accede a información sistematizada (por ejemplo, productos de $I \& D$ de la organización Alter) y a conocimientos de tipo know-how; a formas de resolver problemas y a culturas organizacionales distintas, entre otros aspectos.

Ahuja también identifica a los vínculos indirectos como otro elemento estructural de la red que beneficia el output innovativo de la organización. Los vínculos indirectos son aquellas relaciones entre una organización y otra, que son mediadas por otra organización. El estudio de Ahuja (2000) demuestra que a mayor número de vínculos indirectos, mayor es el subsecuente output innovativo. A modo de síntesis, la evidencia empírica recolectada en diversas investigaciones sostiene un gran cuerpo teórico que hace en extremo difícil negar los numerosos beneficios que conlleva para la organización el posicionamiento en una red de cooperación con otras organizaciones.

\section{IMPLICANCIAS DE LA TEORÍA DE REDES PARA LA FORMACIÓN DE LÍDERES EN EDUCACIÓN}

Es evidente que los propios programas de formación de líderes educativos pueden obtener beneficios a través de potenciar el contacto con otras organizaciones, como, por ejemplo, diversas universidades, otras facultades al interior o exterior de una universidad, organismos públicos como municipalidades o ministerios, escuelas, liceos, empresas privadas, editoriales, organizaciones de investigación y otras. De este modo pueden acceder a la información y a los conocimientos no explícitos que puedan servir al mejoramiento innovativo y constante del programa de formación a través de la adopción de modelos de organización eficaces. Por ejemplo, luego de investigar diferentes modelos de preparación de líderes en Estados Unidos, Grogan y Andrews (2002) recomiendan desarrollar el esfuerzo colaborativo conectando los programas con otras instituciones. Busch y Jackson (2002) sugieren establecer una red internacional que haga posible la transmisión de experiencias para el mejoramiento de estos centros. En su modelo de formación de líderes, Glassman, Cibulka y Ashby (2002) proponen a modo de imperativo la existencia de una red externa que provea de soporte y legitimidad al trabajo facultativo y a los productos de los programas de formación. En este modelo, la red de 
colaboración externa provee al programa de "capacidades de expertos", fuentes de recursos, oportunidades de crecimiento profesional, traspaso de excelencia y oportunidades de influencia individual e institucional (Glassman, N., Cibulka, J., Ashby, D. 2002).

Un buen ejemplo de lo señalado es descrito por Hargador y Sutton (1997) en la investigación etnográfica realizada en la empresa de desarrollo de productos tecnológicos IDEO. Estos autores muestran cómo aquella firma explota su posición de red y sus rutinas internas (orientadas al almacenamiento del conocimiento adquirido gracias a la red) para ayudar a sus ingenieros a crear productos para clientes actuales mediante la combinación de tecnologías previamente existentes, pero que se encontraban dispersas en diferentes organizaciones y, en muchos casos, en distintas industrias. Muchos de esos productos reflejan la transferencia de ideas combinadas nunca antes vistas por las industrias a través de un proceso de "cross pollination" (Hargador, A., Sutton, R. 1997). Transfiriendo estas ideas, la combinación de conocimientos pertenecientes a diversos nichos ofrece la oportunidad a los programas de formación de liderazgo para crear nuevas experiencias innovadoras aplicables a su propio funcionamiento organizacional y al diseño de soluciones educacionales de mayor envergadura. Una forma de concretar esta idea puede ser a través de la utilización de los proyectos de titulación como experiencias de servicio, desarrollo de tecnologías y diseño de estrategias. Con ello, no solo se logra motivar el cierre del proceso formativo, sino también se atienden necesidades reales y problemas relevantes para instituciones educativas específicas ${ }^{1}$.

A través de una política de manejo de redes por parte de los programas de formación en su funcionamiento cotidiano, actuaría un currículo implícito que enseña a operar en redes. Los miembros de las facultades que preparan profesores y los administradores de organizaciones educativas pueden comenzar a contribuir en vistas a un objetivo común. En ambas instancias, no solo se requieren profesores excelentes, sino que también se espera que actúen

1. Esta experiencia ha sido instalada en el programa de Postítulo de Administración de Organizaciones Educativas UC, en el cual los egresados realizan una "Consultoría taller", interactuando con instituciones a las cuales se les proponen soluciones específicas. 
profesionalmente demostrando que la enseñanza excelente va más allá de las actividades lectivas e incluye con potencia el desempeño de los docentes dentro y más allá de la sala de clase (Glassman, N., Cibulka, J., Ashby, D. 2002).

Un estudio adicional (Kelley, C. 2002) concluye que el trabajo colaborativo entre universidad y escuela permite elevar el nivel de calidad del servicio educativo prestado a los estudiantes. Según la autora, el aumento de la calidad del servicio es para las dos instituciones: universidad y escuela. La mantención del vínculo entre egresado y el programa de formación sería, en muchos casos, una buena oportunidad para constituir una alianza sinérgica. Gunter y Ribbins (2002) en su artículo Leadership Studies in Education: Towards a Map of the Field recomiendan que el líder educacional sea apoyado por una universidad o centro de investigación, con lo cual este mantenga actualizado su discurso sobre dirección y apoyo a las prácticas de enseñanza y aprendizaje.

Lo anterior apunta a precisar las ventajas de incluir el conocimiento de manejo de redes en el diseño de programas de formación de liderazgo, si se pretende potenciar el acceso a conocimientos de otras organizaciones y facilitar oportunidades que significarían actuar en posición de "brokering" para la construcción de nuevo conocimiento, con lo que se fortalecería, al mismo tiempo, la eficacia del liderazgo de sus egresados.

\section{PARTICIPACIÓN MULTIDISCIPLINARIA}

Una manera en que un programa de formación de liderazgo puede entrenar al líder en el manejo de la complejidad del entorno es a través de una comprensión y un enfoque holista de la organización (Volante, P., Nussbaum, M. 2002). Este enfoque ayudaría a que las personas en formación sean capaces de integrar áreas diferentes de su formación en la solución de problemas y trabajar interdisciplinariamente. Algunas manifestaciones concretas de un enfoque interdisciplinario en un programa de formación de liderazgo están dadas por la tasa de docentes con diferente formación, experticia y provenientes de culturas profesionales distintas. También, por la tasa de estudiantes seleccionados con formaciones de origen y ocupaciones disímiles, 
además de la diversidad de lenguajes que se utilizan en los cursos y actividades del currículo.

Las implicancias de este enfoque interdisciplinario en el diseño e implementación de programas también pueden transformarse en un plus de calidad para la formación, a pesar de que "sabemos que algunos programas de formación de líderes de formato tradicional desalientan a las personas brillantes que deberían ser atraídas a los cargos de director u otros de liderazgo en educación" (Glassman, N., Cibulka J., Ashby, D. 2002). Por el contrario, se vuelve positiva la existencia de incentivos de ingreso a candidatos de distintas áreas. $\mathrm{Al}$ argumento anterior Glassman, Cibulka y Ashby agregan que la existencia de una mayor diversidad y áreas más amplias en las convocatorias de candidatos implicaría una mayor competencia por el ingreso, lo que podría impactar en una mejor selección.

En vista de lo anterior, algunos de los efectos positivos que acarrearía un enfoque interdisciplinario en la formación de líderes educacionales, son los siguientes:

- Complementariedad y potenciación del posicionamiento de red del programa:

Los miembros del staff de profesores y los estudiantes de diversas áreas cumplen el rol de puentes de acceso a otras organizaciones y áreas de conocimiento para renovar e integrarse a las diferentes culturas educativas. Al mismo tiempo, esta dinámica contribuye a dar forma a un currículo latente de innovación y manejo en red.

- Multidimensionalidad:

La existencia de conocimientos, tanto sistemáticos como también tácitos y culturales, que cruzan diversas disciplinas, puede contribuir a dar forma a un perfil de egresado capaz de comprender los problemas educativos en nuevas dimensiones e integrándolos de manera holista en el diseño de soluciones.

- Innovación:

La fuente amplia de diverso tipo de conocimiento a recombinar que significa el equipo académico multidisciplinario y el grupo de estudiantes que se desempeñan en diferentes áreas ofrece grandes posibilidades de recombinación y de emergencia de nuevo conocimiento innovativo. 
Los planteamientos en torno a considerar el manejo de redes interorganizacionales y la modalidad interdisciplinaria en los programas de formación de liderazgo se orientan a dar forma a un modelo óptimo de formación en el contexto de los desafíos actuales a la gestión educacional, en el supuesto de que los modelos existentes de preparación de líderes educativos requieren de substantivas modificaciones para, a su vez, lograr mejoras substantivas en los sistemas escolares (Young, M., Petersen, P., Short, P. 2002).

\section{EVALUACIÓN DE PROGRAMAS DE FORMACIÓN EN LIDERAZGO EDUCACIONAL}

El estado de la investigación en gestión educacional, y el análisis del discurso político, permiten concluir que en Chile y en muchos otros países ha proliferado la idea y las iniciativas de formación y evaluación de quienes ejercen liderazgo educacional. Existen proyecciones en Estados Unidos que indican que en el año 2005 el setenta por ciento de los directores tradicionales estará en momento de jubilar (Hargreaves, A., Fink, D. 2003). En Chile ya hemos comentado las iniciativas legales en curso y sabemos que el promedio de edad de los directores escolares es más bien alto. Esto nos desafía no solo a pensar en cómo poder inducir una buena formación, sino también a preguntarnos: ¿cómo evaluar los programas de formación de liderazgo en su implementación e impacto posterior?

Un ejemplo a tener en cuenta para valorar la potencia de los programas de formación que buscan desarrollar competencias de liderazgo educacional, fue elaborado como un sistema de autoevaluación y mejoramiento continuo en Estados Unidos, donde abundan este tipo de programas, por Glassman, Cibulka y Ashby (2002). Estos autores afirman que los miembros de las instituciones de formación de educadores y los administradores de las organizaciones educativas deberían conversar más y compartir iniciativas para evaluar y mejorar en sus objetivos comunes. Con esta idea, estos autores recomiendan la construcción de una estructura de mejoramiento continuo que se apoya en dos tareas base: la creación de un ambiente de aprendizaje coherente y el alineamiento del currículo e instrucción en torno a un eje basado en capacidades centrales. 
Ambas tareas pueden ser facilitadas con modelos de estándares de desempeño, con proyectos en red entre instituciones formadoras y usuarias, y con la incorporación de formadores de distintas culturas organizacionales pero muy vinculadas a la generación de líderes.

A modo de ejercicio inicial, a continuación se propone una serie de preguntas en torno a esta pretensión, las cuales han sido adaptadas desde el estudio de Glassman, Cibulka y Ashby, y pueden servir de orientación no solo para quienes están trabajando para mejorar la formación de líderes en educación, sino también para quienes desean invertir en este tipo de entrenamientos.

1. ¿Aporta el programa herramientas para articular una visión de largo plazo apoyada por la comunidad escolar?

2. ¿Brinda mecanismos para la implementación, evaluación y monitoreo de la visión?

3. ¿Ofrece herramientas para desarrollar símbolos y ritos observables que caractericen a la visión de la institución educativa?

4. ¿Aporta conocimientos para generar una cultura organizacional centrada en el aprendizaje escolar?

5. ¿Otorga instrumentos para la administración y gestión de operaciones eficaces?

6. ¿Provee conocimientos para poder desarrollar proyectos que involucran esfuerzos conjuntos y de cooperación?

7. ¿Ofrece mecanismos para movilizar recursos de la comunidad?

8. ¿Aporta herramientas para cubrir las necesidades e intereses de la comunidad?

9. ¿Ayuda a comprender los elementos y procesos externos que tienen efectos significativos en la vida de la institución?

10. ¿Ofrece herramientas para ejercer una relativa influencia sobre el contexto político, legal, económico, social y cultural?

11. ¿Brinda instrumentos para establecer una red interescolar o interorganizacional que signifique beneficios para la institución educativa?

12. ¿Aporta un marco ético para tomar y comunicar claramente decisiones íntegras y justas?

La idea de impulsar evaluaciones sistemáticas y procesos de mejoramiento continuo para estos programas supone que "una efectiva evaluación llevará a alcanzar mejores niveles en la formación de líderes, 
y a mejorar sus prácticas subsecuentes" (Glassman, N., Cibulka J., Ashby, D. 2002). Este marco de evaluación se encuentra enfocado principalmente al incremento de logros educacionales, y se relaciona bastante con los Estándares para el Liderazgo Educacional del tipo ISLLC (1996). En ambos referentes prima una visión de liderazgo centrada en las acciones que contribuyen a fortalecer los resultados de aprendizaje, independiente del rol formal que se ejerza. Lo cual pone en cuestión la tradición de diseñar programas de postgrado o postítulo destinados a preparar para cargos o funciones administrativas específicas (director, jefe de unidad técnica pedagógica, orientador, entre otros). El cumplimiento de estos criterios se relaciona con una formación interdisciplinaria que permite una comprensión global de las diferentes tareas del líder educacional, y con ello, los alcances de su propósito principal: hacer que los estudiantes, profesores y otros miembros se concentren en lograr aprendizajes de calidad.

\section{ALGUNAS IDEAS CLAVES}

A modo de síntesis se señalan las siguientes ideas que destacan lo expuesto:

Existen diferencias conceptuales entre liderazgo, gestión y administración, que son relevantes para definir perfiles de formación de líderes en educación.

Hay un currículo global basado en estándares que influye en los programas de formación de liderazgo en educación.

En los diferentes estándares y programas de formación de liderazgo en el mundo se encuentran alineados tanto los conocimientos necesarios como las competencias básicas para alcanzar buenos resultados académicos y contribuir al mejoramiento de la organización educativa.

La confluencia de los conocimientos interdisciplinarios y la capacidad de posicionar a la organización educativa dentro de una red interorganizacional se constituyen como dos nuevas herramientas para mejorar las prácticas del líder educativo. 


\section{ReFERENCIAS BibLIOGRÁFICAS y NOTAS}

Acuña, Eduardo. "Los peligros del liderazgo efectivo". Estudios Sociales. $\mathrm{N}^{\mathrm{o}} 80$, trimestre 2, 1994.

Ahuja, Gautam. Collaboration Networks, Structural Holes and Innovation: a Longitudinal Study". Administrative Science Quarterly. Vol. 49, 2000.

Busch, Toni; Jackson, David. A Preparation for School Leadership International Perspectives. Educational Management \& Administration. Vol. 30, número 4, 2002.

Cambron-Mc Cabe, Nelda; Cunningham, Lubern. National Commission for the Advancement of Educational Leadership. Educational Administration Quarterly. Vol. 38, número 2, 2002.

Clark, Jane. Editor's Note. Ensuring the Capacity of University-Based Educational Leadership Preparation: The Collected Works of the National Commission for the Advancement of Educational Leadership Preparation. Educational Administration Quarterly. Vol. 38, No 2, 2002.

Day, Christopher. Beyond transformation leadership. Educational Leadership. Vol. 57, No 7, 2000.

Di Maggio, Paul. Nadel Paradox Revisited: Relational Anit Cultural Aspects of Organizational Structure. Nohria and Eccles. Networks and Organizations: Structure Form and Action. Boston Harvard Business School Press, 1992.

Glassman, Naftaly; Cibulka, Jane \& Ashby Dianne. Program Selfevaluation for Continuous Improvement. Educational Administration Quarterly. Vol. 38, No 2, 2002.

Grogan, Margaret \& Andrews, Richard. Defining Preparation and Professional Development for the Future. Educational Administration Quarterly. Vol. 38, No 2, 2002.

Gunter, Helen \& Ribbins, Peter. Leadership Studies in Education, Towards a Map of the Field. Educational Management \& Administration. Vol. 30, No 4, 2002. 
Hargador, Andrew; Sutton, Robert. Technology Brokering and Innovation a Product Development Firm. Administrative Science Quarterly. No 42, 1997.

Hargreaves, A.; Fink, D. Sustaining Leadership. Phi Delta Kappan, mayo 2003.

Heifetz, R. A. Leadership Without Easy Answers. Cambridge, MA: Harvard University Press, 1994.

Huber, S.G., West, M. Developings Schools Leaders: A Critical Review of Current Practices, Approaches and Issues and Some Directions for the Future. Second International Handbook of Educational Leadership and Administration, 1071-1101. K. Leithwood, P. Hallinger (eds.). Kluwer International Handbooks of Education, 2002.

ISLLC, Standards For School Leaders. Council of Chief State School Officers, 1996.

Jackson, Barbara; Kelley, Carolyn. Exceptional and Innovative Programs in Educational Leadership. Educational Administration Quarterly. Vol. 38, No 2, 2002.

Kelley, Carolyn. Politics, Community and Leadership in the Relationship between Universities and Schools. Educational Administration Quarterly. Vol. 38, No 4, 2002.

Leithwood, K.; Jantzi, D.; Steinbach, R. Leadership Practices for Account Schools. Second International Handbook of Educational Leadership and Administration. Kluwer International Handbooks of Education, 2002 .

Marsden, Peteer. Broker Behavior in Restricted Change Network. Marsed and Lin. Social Structure and Network Analysis, 1992.

Marshall, Catherine. Call for Proposals: Leadership for Social Justice and Equity. Educational Administration Quarterly. Vol. 38, N 2, 2002.

Montt, Pedro. El mejoramiento pedagógico y la gestión escolar son dos caras de la misma moneda. Entrevista en Revista de Educación No 298, septiembre, 2002: pp. 32-34. 
Mulford, Bill. The Global Challenge a Matter of Balance. Educational Management \& Administration. Vol. 30, No 2, 2002.

Murphy, Joseph. Reculturing the Profession of Educational Leadership. Educational Administration Quarterly. Vol. 38, No 2, 2002.

Petersen, Kent. The Professional Development of Principals: Innovations and Opportunities. Educational Administration Quarterly. Vol. 38, $\mathrm{N}^{\mathrm{o}} 2,2002$.

Podolny, Joel; Page, Karen. Network Forms of Organization. Annual Review of Sociology. Vol. 24: 57-76. 1998.

Powel, W.; K. W. Koput; L. Smith-Doerr. Interorganizational Collaboration and the Locus of Innovation: Networks of Learning in Biotechnology. Administrative Science Quarterly. Vol. 41, 116-145. 1996.

Randall, Hollenbeck; Stone. Teacher Performance Incentives and Student Outcomes. The Journal of Human Resources. Vol. 37, No 4, 2002.

Rodríguez, Darío. Gestión organizacional. Elementos para su estudio. Santiago: Ediciones Universidad Católica, 2001.

Shan, W.; G. Walker; Kogut, B. Interfirm Cooperation and Startup Innovation in the Biotechnology Industry. Strategic Management Journal. Vol. 15, 387-394. 1994.

Spillane, James; Halverson, Richard; Diamond, John. Investigating School Leadership Practice, a Distributed Perspective. Educational Leadership, 2002.

Usdan, Michael. Reactions to Articles Commissioned by the National Commission for the Advancement of Education Leadership Preparation. Educational Administration Quarterly. Vol. 38, Nº 2, 2002.

Volante, Paulo; Castro, Pablo; Isla, Pablo; Müller, Magdalena. Estándares para el liderazgo educativo. Boletín de investigación educacional. Facultad de Educación, Pontificia Universidad Católica de Chile, volumen 17, 2002. 
Volante, P.; Nussbaum, M. Cuatro principios de acción en gestión educacional. Revista de Ingeniería de Sistemas. Universidad de Chile, Vol. XVI, No 1 Junio 2002.

Young, Michelle; Petersen, George. Guest Editors Introduction. The National Commission for the Advancement of Educational Leadership Preparation: An Introduction. Educational Administration Quarterly. Vol. 38, No 2, 2002.

Young, Michelle D.; Petersen, George J.; Short, Paula M. "The Complexity of Substantive Reform: A Call for Interdependence Among Key Stakeholders". Educational Administration Quarterly. Vol. 38, $\mathrm{N}^{\mathrm{o}} 2,137-175.2002$. 
